\title{
DESAJUSTES EN EL MERCADO DE TRABAJO E INFLACIÓN: UN MODELO INTEGRADOR ESTIMADO PARA EL CASO ESPAÑOL DURANTE LA GRAN RECESIÓN ${ }^{1}$
}

\author{
MISMATCH IN THE LABOR MARKET AND INFLATION: AN INTEGRATIVE MODEL \\ ESTIMATED FOR THE SPANISH CASE DURING THE GREAT RECESSION
}

\author{
DESAJUSTES NO MERCADO DE TRABALHO E INFLAÇÃO: UM MODELO DE \\ INTEGRAÇÃO ESTIMADO PARA O CASO ESPANHOL DURANTE A GRANDE \\ RECESSÃO
}

\author{
Santos M. Ruesga² \\ Laura Pérez Ortiz 3 \\ Friedrich L. Sell ${ }^{4}$
}

\begin{abstract}
Resumen
La Gran Recesión ha enfatizado la necesidad de diferentes políticas económicas y reformas estructurales para impulsar el crecimiento económico y la contratación de las economías avanzadas y emergentes. Partimos del concepto teórico relativamente novedoso: la brecha de producción modificada (MOG), basada tanto en la curva de Phillips como en la de Beveridge, que revela la relación explícitamente positiva entre la tasa de vacantes de empleo y la tasa de inflación. Empíricamente se estima la relación mediante el desarrollo de tres modelos de datos de panel diferentes: de efectos fijos (FE), de efectos aleatorios (RE) y un sistema GMM (Método
\end{abstract}

DOi: https://doi.org/10.15359/eys.23-54.6

Fecha de recepción: 28-08-2018. Fechas de reenvíos: 19-10-2018, 30-10-2018. Aceptado el 9-11-2018. Publicado el 27-11-2018.

${ }^{1}$ Queremos agradecer su colaboración a Manuel Pérez Trujillo, actualmente docente en la Universidad Católica del Norte, de Chile, quien en versiones preliminares de este trabajo participó en su elaboración, particularmente en los aspectos metodológicos aplicados. Asimismo, agradecemos el trabajo del equipo evaluador anónimo, que con sus comentarios ha contribuido a mejorar de forma notable la calidad de este artículo.

2 Doctor en Economía, catedrático de Economía Aplicada en el Departamento de Estructura Económica y Economía del Desarrollo, de la Universidad Autónoma de Madrid (UAM), España. Correo electrónico ruesga@uam.es

${ }^{3}$ Doctora en Economía, profesora ayudante en el Departamento de Estructura Económica y Economía del Desarrollo de la Universidad Autónoma de Madrid (UAM), España. Correo electrónico laura.ortiz@uam.es

${ }^{4}$ Doctor en Economía, catedrático de Macroeconomía y Política Económica en la Universitat der Bundeswehr München, Alemania. Correo electrónico friedrich.sell@unibw.de

74

Santos M. Ruesga, Laura Pérez Ortiz y Friedrich L. Sell

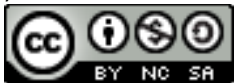

Revista Economía y Sociedad by Universidad Nacional is licensed under a CreativeCommons Reconocimiento-NoComercialCompartirlgual 4.0 Internacional License. 
generalizado de momentos). Los resultados muestran que una pérdida de la eficiencia en el ajuste en el mercado laboral combinada con un aumento de la demanda en los mercados de bienes y servicios elevará la inflación. Se evidencia la relevancia empírica de la brecha de producción modificada para España durante la Gran Recesión y cómo afecta la implementación del plan de estímulo económico liderado por el gobierno español para impulsar la economía en este período.

Palabras claves: Curva de Beveridge; curva de Philips; desajuste laboral; inflación; mercado de trabajo

\begin{abstract}
The Great Recession has emphasized the need for different economic policies and structural reforms to boost economic growth and advanced emergent economies. This paper is founded on a relatively innovative theoretical concept: the Modified Output Gap (MOG). Based on the Phillips and Beveridge curves, the MOG reveals an explicitly positive relationship between the job vacancy rate and the inflation rate. Empirically, the relationship is estimated by developing three different panel data models: Fixed Effects (FE), Random Effects (RE) and Generalized Method of Moments (GMM). Results shows that a mismatch in the efficiency of the labor market combined with an increased demand in goods and services markets will increase inflation. This paper shows the empirical relevance of the modified output gap for Spain during the Great Recession and how it affects the implementation of the economic stimulus plan led by the Spanish government to boost the economy in this period.
\end{abstract}

Key words: Beveridge curve; Phillips curve; labor mismatch; inflation; labor market

\title{
Resumo
}

A Grande Recessão enfatizou a necessidade de diferentes políticas econômicas e reformas estruturais para impulsionar o crescimento econômico e a contratação de economias avançadas e emergentes. Partimos do conceito teórico relativamente novo: a brecha de produção modificada (MOG), baseado tanto na curva de Phillips quanto na de Beveridge, revelando a relação explicitamente positiva entre a taxa de vagas do emprego e a taxa de inflação. Empiricamente, a relação é estimada através do desenvolvimento de três modelos de dados de painel diferentes: efeitos fixos (FE), efeitos aleatórios (RE) e um sistema GMM (método generalizado dos momentos). Os resultados mostram que uma perda da eficiência no ajuste no mercado de trabalho combinada com um aumento na demanda nos mercados de bens e serviços aumentará a inflação. A relevância 
empírica da lacuna de produção modificada na Espanha durante a Grande Recessão é evidente, e como isso afeta a implementação do plano de estímulo econômico liderado pelo governo espanhol para impulsionar a economia neste período.

Palavras-chave: Curva de Beveridge; curva de Philips; desajuste laboral; inflação; mercado de trabalho

\section{Introducción}

La Gran Recesión (2007-2013) ha dado lugar a muchas divergencias en el comportamiento de las economías desarrolladas. También ha enfatizado la necesidad de llevar a cabo diferentes políticas económicas y reformas estructurales en dichas economías. Durante este período, el mercado laboral sufrió un impacto negativo, lo cual llevó a que los entes responsables de las políticas implementaran diversas propuestas económicas que permitieran recuperar el nivel de empleo registrado antes del comienzo de la Gran Recesión, tratando, entre otros objetivos instrumentales, de mejorar la eficiencia del ajuste entre la oferta y la demanda en el mercado laboral.

A la hora de evaluar el impacto de estas propuestas de política económica resulta preciso acudir al empleo de herramientas adecuadas. Con tal finalidad, en este artículo, se utilizan dos herramientas teóricas: la curva de Beveridge $(B C)^{5}$ (Beveridge, 1944 ) y la curva de Phillips $(P C)^{6}$ (Phillips, 1958).

El uso de ambas herramientas puede permitir determinar teóricamente cómo el shock negativo introducido por la Gran Recesión en la economía puede haber elevado el nivel de desempleo estructural en el mercado laboral.

Se han combinado ambas herramientas para generar el concepto teórico de brecha de producción modificada (MOG, por sus siglas en inglés, modified output gap) (Sell, \& Reinisch, 2013). El marco analítico de la MOG facilita la comprensión de la ineficacia de las políticas económicas cuando hay un shock negativo que afecta la eficiencia del ajuste en el mercado laboral, también conocido como desajuste ("mismatch", ver Petrongolo y Pissarides, 2001), lo que aplicaremos a un análisis empírico para confirmar tales hipótesis en el caso español.

En esta perspectiva, el presente estudio se centra en el primer periodo de la recesión económica en España, entre 2007 y 2010. Tanto el país como el período son apropiados para

\footnotetext{
${ }^{5}$ La BC es una herramienta teórica útil para analizar el mercado laboral en presencia de fricciones que afectan su equilibrio. Es una función que representa el número de empleos ocupados en un momento dado en relación con el número de personas que buscan empleo (desempleo), el número de empresas que buscan trabajadores y trabajadoras (vacantes abiertas) y un pequeño conjunto de otras variables (Petrongolo y Pissarides, 2001). Suponiendo que la curva está en un estado estacionario, en el que la variación en el número de vacantes y personal empleado de un período a otro es 0 (dado que el flujo de entrada y salida del desempleo es constante), el equilibrio de la curva se caracteriza por una relación de elasticidad negativa entre vacantes y desempleo.

${ }^{6}$ La PC, llamada así por el economista neozelandés William Phillips (1958), establece la conocida relación negativa entre el desempleo y la inflación.
}

76

Santos M. Ruesga, Laura Pérez Ortiz y Friedrich L. Sell 
probar la relevancia empírica del MOG. Son pocos los estudios recientes que han demostrado la pérdida de eficiencia en el ajuste en el mercado laboral español después del comienzo de la crisis económica (European Commission, 2012; Haincourt \& Mogliani, 2012; Hobijn \& Sahin, 2012; Organización para la Cooperación y el Desarrollo Económico (OCDE), 2013; Ruesga, Silva \& Pérez, 2015; Wölfl \& Mora-Sanguinetti, 2011). La mayoría de estos grupos asocian esta situación al estallido de la burbuja inmobiliaria y al colapso del modelo de producción que caracterizó la expansión de la economía antes del período de crisis. Debido a la recesión y al rápido aumento del desempleo al comienzo de la crisis, el gobierno español liderado por Zapatero decidió adoptar un plan de estímulo económico, conocido como Plan E, que se aplicó entre noviembre de 2008 y el primer semestre de 2010, con el fin de impulsar la economía y el empleo.

Sin embargo, el impacto general de este plan económico fue limitado. Por un lado, no pudo detener, a lo largo de su implementación, la pérdida progresiva de empleos y el creciente desempleo, especialmente en los sectores más afectados por la crisis. Por otro lado, conviene tener presente que las empresas donde la demanda laboral aumentó durante el período de aplicación de este plan económico se concentraron, básicamente, en el sector de servicios -educación, salud, administración pública, y suministro de electricidad, gas, vapor y aire acondicionado-. Al mismo tiempo se registró un aumento simultáneo en las horas extraordinarias de trabajo, a causa de la carencia de personal adecuado disponible en el mercado para ocupar las nuevas vacantes generadas en las actividades económicas en expansión. Situación que favoreció el ascenso de los salarios en estos sectores y afectó, en el mediano plazo, los costos de producción e introdujo, así, tensiones importantes para el mantenimiento del nivel de inflación en toda la economía.

En general, el Plan E se convirtió en un ejemplo de una política económica mal diseñada que impulsó la demanda en el mercado de bienes y servicios, sin corregir el desequilibrio entre la oferta y la demanda en el mercado laboral, poniendo en riesgo el equilibrio macroeconómico ${ }^{7}$. ${ }^{7}$ Como alternativas al plan de estímulo económico "fallido", desarrollado por el gobierno español en 2009-2010,
se han llevado a cabo numerosos planes de reformas cuyas estructuras apuntan a flexibilizar el mercado laboral.
Estos planes son similares a los implementados en otros países afectados por la crisis de 2008, principalmente
en la Eurozona. El más significativo lo constituye la reforma laboral introducida en 2012, por el gobierno
conservado de Mariano Rajoy. La principal consecuencia de esta reforma ha sido una disminución en los costos
laborales que permitió la economía española para ganar competitividad en el corto plazo en relación con sus
competidores en mercados internacionales y así mejorar el nivel de exportaciones y empleo, al menos
ralentizando la disminución en el nivel de empleo (Lacuesta \& Puente 2013 ; OCDE, 2013). Pero la efectividad de
este tipo de reformas es limitada en el mediano a largo plazo con respecto a la creación de empleo. En un
contexto económico en el que todos los socios comerciales realizan reformas similares (como las aplicadas en
otros países de la UE a las que se exporta más del $60 \%$ de todas las mercancías y servicios españoles), orientadas
a reducir los costes laborales, con lo cual influyen principalmente en el nivel salarial y en la capacidad de gasto y
consumo: esta política provoca una reducción en la demanda externa que socava los beneficios obtenidos por
las ganancias previas de competitividad; afectan, así, el crecimiento de la economía y el desempleo. Por lo tanto,
es crucial llevar a cabo planes de estímulo monetarios y fiscales (especialmente fiscales en países con superávit
a nivel comercial), ya que dichos planes que garanticen una demanda sostenida y permitan restaurar el nivel de
crecimiento económico y empleo alcanzados antes de la crisis en los países más afectados (Capaldo \& Izurieta
2013 ). Por lo tanto, este artículo no pretende criticar la efectividad de los programas de estímulo económico
para resolver la crisis económica, sino que muestra los problemas adicionales que deberían evaluarse, como el

Santos M. Ruesga, Laura Pérez Ortiz y Friedrich L. Sell 
En este contexto, claramente se pone de manifiesto la importancia de comprender las implicaciones teóricas del enfoque MOG.

El artículo se organiza de la siguiente manera. En primer lugar, se analiza e integra tanto la BC como la PC, para obtener de estos dos conceptos el MOG. Una vez aclarado el devenir teórico de estas herramientas, se describe el desempeño de la economía española, centrando el análisis en el mercado laboral durante la Gran Recesión y en el efecto del plan de estímulo económico desarrollado por el Gobierno para situar a la economía en la senda del crecimiento. Y a continuación, se prueba empíricamente el comportamiento de BC, PC y MOG durante la crisis para el caso español. Al final del documento, se señalan las principales conclusiones extraídas, tanto en el plano teórico como en el análisis empírico realizado, para extraer algunas implicaciones de política económica a este respecto.

\section{La brecha de producción modificada (MOG)}

La BC y la PC son dos herramientas teóricas comúnmente utilizadas por economistas durante el siglo pasado para comprender los desequilibrios del mercado laboral. Se combinan estos dos conceptos teóricos para obtener la relación a la que nos referimos como MOG (Sell \& Reinisch, 2013) ${ }^{8}$. La lógica de esta relación puede explicarse de la siguiente manera: cuando el mercado laboral está en expansión, el número de empleos disponibles (vacantes) crece y el desempleo disminuye, siguiendo la lógica de la BC. Y, simultáneamente, cuando el desempleo disminuye y la demanda agregada se fortalece, el nivel de precios tiende a aumentar, siguiendo la lógica establecida por la PC. Debido a ambos procesos, se observa una relación positiva entre las vacantes de empleo y la inflación dentro del equilibrio macroeconómico general, según enfoque del MOG (Sell, 2010).

Por lo tanto, es importante distinguir entre los movimientos a lo largo de la curva que representa el MOG y los cambios que afectan su estabilidad (ver Figura 1). La idea de un cambio en la estabilidad de la función es bastante simple. Por un lado, el deterioro de la eficiencia en el ajuste del mercado laboral empuja a la BC hacia el exterior del eje de coordenadas $^{9}$, de modo que se mueve desde la posición de equilibrio a, sobre $\mathrm{BC}^{0}$, al equilibrio definido por $\mathrm{b}$ ubicado sobre $\mathrm{BC}^{1}$, en un movimiento indicado por la flecha 1 (ver cuadrante I). Este deterioro de la eficiencia o el aumento del desajuste tiene consecuencias a corto y mediano plazo para la estabilidad del MOG. El efecto inmediato de la menor eficiencia en el ajuste y el descenso de la demanda laboral se traduce en el aumento del desempleo, lo que deprime, por tanto, la demanda agregada. Todo ello que conduce a una disminución de los precios en el corto y mediano plazo. Este resultado desplaza $\mathrm{MOG}^{0}$ a $\mathrm{MOG}^{1}$, cambiando el

\footnotetext{
empeoramiento de la eficiencia en el ajuste entre la oferta y la demanda en el mercado laboral, con el propósito de asegurar que las políticas se apliquen y desarrollen adecuadamente.

${ }^{8}$ Véase también Sell (2010) para una mejor comprensión del desarrollo matemático del MOG.

${ }^{9}$ La bibliografía económica relaciona las ganancias o pérdidas de la eficiencia en el ajuste con el avance tecnológico, la divergencia entre el nivel de educación de las personas trabajadoras y la capacitación requerida por las empresas, la movilidad del factor de trabajo, el costo de los despidos y el modelo de políticas pasivas de mercado laboral (Blanchard \& Diamond, 1989; Petrongolo \& Pissarides, 2001).
}

78

Santos M. Ruesga, Laura Pérez Ortiz y Friedrich L. Sell 
equilibrio de $a^{\prime \prime}$ a $b^{\prime \prime}$, en la dirección de la flecha 1 , en el cuadrante IV, donde la relación inflación-vacantes es menor.

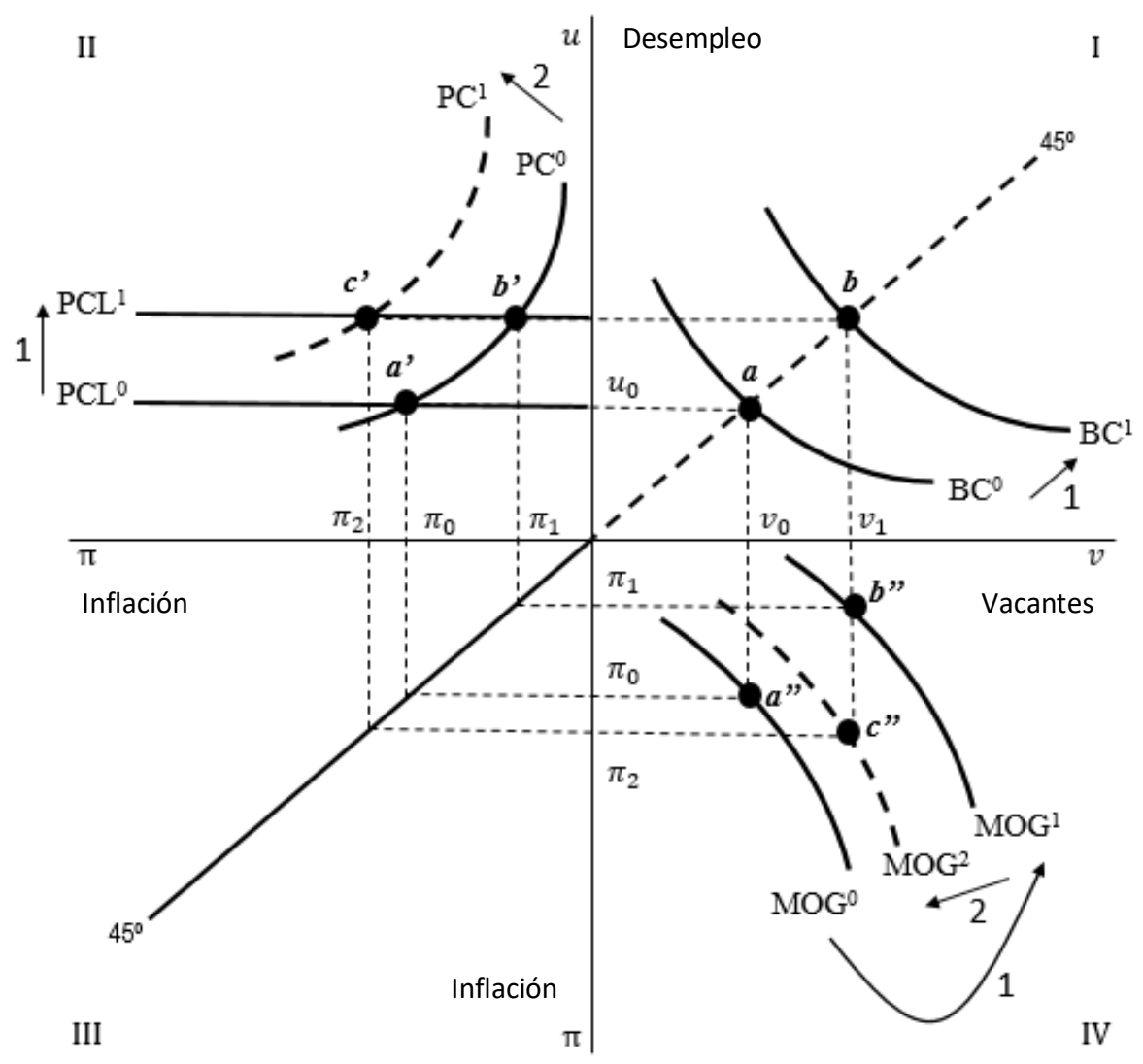

Figura 1. Efecto de la pérdida de eficiencia del mercado laboral sobre la curva MOG. Fuente: elaboración propia.

Sin embargo, a largo plazo, como indican Kaufman \& Hotchkiss,

La llamada tasa natural de desempleo ... está dada por la intersección entre la BC y la línea de 45 grados, donde el número de solicitantes de empleo (desempleados)/vacantes $(u)$ es igual a el número de ofertas de empleo/vacantes (v) y así el mercado laboral está en equilibrio a largo plazo. (2006, p. 217)

Por lo tanto, cualquier cambio en la BC moverá la PC a largo plazo, desplazándolo desde el equilibrio $a^{\prime}$ en $\mathrm{PCL}^{0}$ a $b^{\prime}$ en $\mathrm{PCL}^{1}$, como lo indica la flecha 1 en el cuadrante II. La transformación que se produce en el mercado laboral afectará la tasa de inflación a largo plazo, y generando un cambio positivo en el corto y medio plazo de la PC, en tanto que los agentes anticipan el futuro incremento del nivel de precios; mientras que también la PC se desplaza a la derecha del eje de coordenadas, trasladando el equilibrio de $b^{\prime}$ sobre $\mathrm{PC}^{0}$ a $\mathrm{c}^{\prime}$ sobre PC $^{1}$, tal como señala la flecha 2 del cuadrante II. Este segundo movimiento de la PC provocará que el MOG se mueva hacia adentro, de b" en $\mathrm{MOG}^{1}$ a c" en $\mathrm{MOG}^{2}$, como lo indica 
la flecha 2 del cuadrante IV, lo que indica un nivel de inflación más alto para cada nivel de vacantes en el mercado, ceteris paribus ${ }^{10}$.

Por otro lado, el doble impacto de la política de estímulo ya sea fiscal o monetario, en el equilibrio del MOG es la siguiente. Por un lado, suponemos que el gobierno decide actuar sobre la economía en un momento desfavorable para estimular el crecimiento y, por lo tanto, el empleo. Según la dinámica de la Figura 2 , la situación descrita desplazaría la $\mathrm{PC}$ de $\mathrm{PC}^{0}$ a $\mathrm{PC}^{1}$, trasladando así el equilibrio de $\mathrm{a}^{\prime}$ a b' $\mathrm{b}^{\prime}$, donde el nivel de desempleo es más bajo, pero los precios aumentan (véase el cuadrante II). La curva cambia debido a que el aumento en la oferta no compensa el impulso de la demanda agregada generada por el plan de estímulo, creando expectativas de mayor inflación, lo que hace que las PC cambien positivamente en el corto plazo (Sell, 2010).

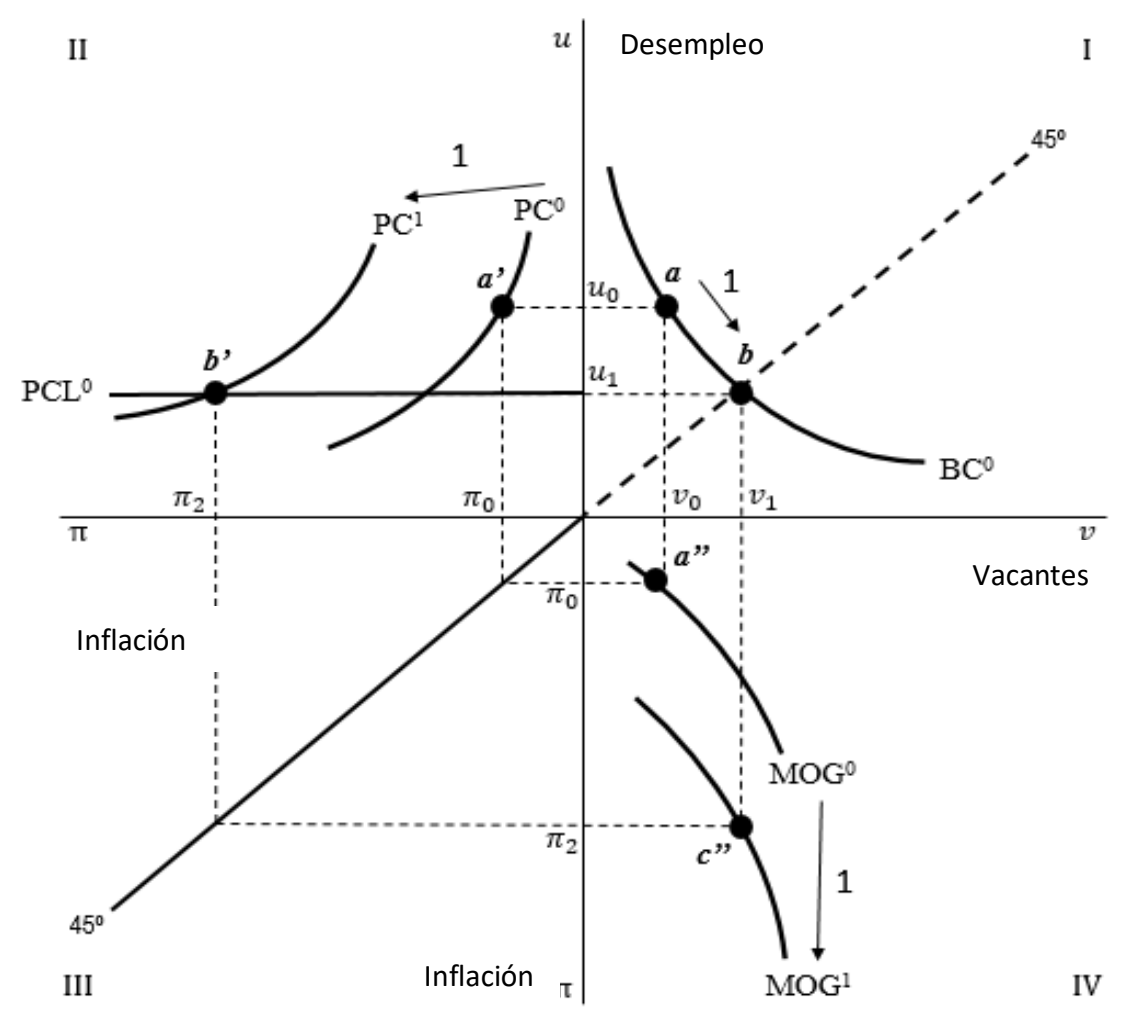

Figura 2. Efecto de una política de estímulos sobre el equilibrio de la MOG.

Fuente: elaboración propia.

Debido a la dinámica que se ha producido en la PC, la curva MOG también cambiará, moviéndose hacia adentro desde $\mathrm{MOG}^{0}$ a $\mathrm{MOG}^{1}$. Cualquier cantidad de vacantes ahora se acompañará de tasas de inflación más altas, un equilibrio determinado por c".

\footnotetext{
${ }^{10}$ La estabilidad de MOG se verá afectada por ambos movimientos, primero, de la BC y, segundo, de la PC. Cambiará hacia adentro, una relación de vacantes de inflación peor o externa que mejorará la relación, dependiendo de si la intensidad del movimiento de la BC o de la PC ejerce una influencia más fuerte sobre el MOG o sobre la elasticidad de ambas curvas.
}

80

Santos M. Ruesga, Laura Pérez Ortiz y Friedrich L. Sell 
La combinación teórica de BC y PC en el contexto analítico del MOG nos proporciona una mejor comprensión de otras ineficiencias en la política macroeconómica cuando hay un shock negativo que afecta la eficiencia de los partidos en el mercado laboral. Cualquier impacto positivo en la economía, como el resultado de un extenso programa de gasto público para aumentar la demanda agregada, será mucho menos efectivo, dada la baja eficiencia en el mercado laboral. La demanda agregada adicional dará como resultado un aumento en el nivel de inflación. ¿Por qué? Las empresas no pueden contratar (personal calificado y menos calificado, ya que no hay heterogeneidad en términos de habilidades y ningún proceso de negociación explícito en el modelo MOG) suficientes trabajadores y trabajadoras para aumentar su nivel de producción de acuerdo con el nuevo nivel de demanda. En consecuencia, tendrán que usar su dotación de factor de trabajo existente más intensamente, lo que eventualmente aumentará los costos de producción. En un nivel significativo de competencia monopolística, las empresas pasarán estos altos costos de producción a la demanda final.

\section{La economía española durante la gran recesión: Mala previsión y escasos resultados de las medidas de expansión fiscal ${ }^{11}$}

En esta sección, se analiza la evolución de la economía española -el tema principal de esta investigación para demostrar la relevancia empírica del MOG-y el mercado laboral durante el comienzo de la recesión económica. Con el estallido de la burbuja inmobiliaria y el colapso del modelo económico imperante durante el período de crecimiento de la economía entre 1995 y finales de 2007, se produjeron cambios importantes que afectaron negativamente al mercado laboral.

En el período comprendido entre el cuarto trimestre de 2007 y el segundo de 2010 (último trimestre analizado), el desempleo experimentó un aumento mucho mayor en términos relativos, alrededor de 1,4 veces durante este período, alcanzando a 4 645,5 miles de desempleados al final de este, comparado con la disminución observada para el número de vacantes que cayó solo al 4,5 por ciento. El diferente ritmo de variación tanto de la tasa de desocupación como de desempleo puede indicar la existencia de fricciones que afectaron la falta de coincidencia durante la crisis.

Un análisis de la evolución de la estructura del empleo podría ayudar a comprender lo que ocurrió en el mercado laboral durante este período. En este sentido, la expansión de la intensidad de la tecnología baja y media baja, y las industrias del conocimiento durante el crecimiento económico y después de la recesión en estos sectores explican la desaceleración en el mercado laboral. Entre 2001 y 2007, el empleo creció alrededor de 4,6 millones, donde el sector de la construcción por sí solo creó el 20 por ciento del empleo total. La actividad económica en esta fase fue impulsada principalmente por industrias con mano de obra intensiva que ocupaba personal con un nivel de calificación bajo y medio bajo (Ruesga, Silva, \& Pérez, 2015).

\footnotetext{
${ }^{11}$ Los datos de esta sección sobre el nivel de empleo y desempleo se obtienen de la Encuesta de Población Activa (EPA), del INE, mientras que los datos de vacantes provienen de la Encuesta Trimestral de Coste Laboral (LCI), elaborado por el Ministerio de Trabajo y Seguridad Social, ambos españoles.
} 
Sin embargo, cuando comenzó la recesión, estas industrias contribuyeron más para la disminución del empleo total, por ejemplo, en el sector de la construcción el 52,5 por ciento del total de empleos se redujo entre el primer trimestre de 2008 y el segundo de 2010. A medida que avanzaba la recesión, el personal ocupado en industrias menos competitivas fue el más afectado por el proceso de destrucción de empleo, debido a su baja empleabilidad (Bell \& Blanchflower, 2011; Wölfl \& Mora-Sanguinetti, 2011).

Para mitigar el impacto negativo de la recesión en el mercado laboral al inicio de la crisis, el gobierno español decidió aplicar un plan de estímulo económico conocido como Plan de Estímulo de la Economía y el Empleo (Plan E) (ver Real Decreto-Ley 9/2008)). Este plan funcionó desde noviembre de 2008 hasta la segunda mitad de 2010 y tuvo un monto aproximado de $€ 11000$ millones, que abarca 80 medidas económicas, financieras y fiscales para impulsar el crecimiento económico y la creación de empleo.

El Plan E inicialmente favoreció la creación de empleo, similar a los planes económicos de estímulo realizados al comienzo de la recesión por otros países de la OCDE (Capaldo, Izurieta, 2013) y el aumento de las vacantes disponibles en el mercado laboral en un $10.6 \%$ entre el cuarto trimestre de 2008 y el segundo de 2010. Además, este plan redujo la tasa de crecimiento del desempleo; el cual, sin embargo, aumentó en un $44,8 \%$ en el período. En este sentido, las industrias que más contribuyeron a la destrucción del empleo al comienzo de la recesión continuaron con el proceso de ajuste; donde nuevamente el sector de la construcción estaba en la cima con el $37,1 \%$ del empleo total disminuido.

Además, el crecimiento de las vacantes experimentadas durante el plan de estímulo económico se concentró en el sector de servicios. En este sector, el aumento en las vacantes fue del 24,6 \%. Este proceso impulsó el empleo en sectores como la educación, los servicios de salud, la administración pública y la electricidad, el gas, el vapor y el suministro de aire acondicionado. Tales sectores compensaron la caída general del empleo durante este período con la creación de más de 144300 nuevos empleos, mientras que al mismo tiempo aumentó el número de horas trabajadas (ver Tabla 1). En este sentido, ambos valores indican que la demanda laboral creció en estos sectores durante el plan de estímulo.

La mayor demanda en los sectores que requieren mano de obra calificada o un nivel específico de capacitación contrasta con el aumento simultáneo del desempleo. Esta demanda se reforzó con un ascenso de los sectores laborales intensivos en trabajo, cuya plantilla se caracterizaba por un nivel de calificación bajo y medio bajo. En este sentido, la dificultad para quienes tenían un bajo nivel de empleabilidad para encontrar un nuevo empleo, en los sectores que demandaban nuevos empleados, podría haber afectado el desajuste en el mercado laboral, y el nivel de desempleo estructural (Bouvet, 2011). 


\section{Tabla 1.}

Variación del tiempo de trabajo por persona trabajadora ocupada a tiempo completo $y$ empleo, durante el período comprendido entre el cuarto trimestre de 2008 y el segundo trimestre de 2010

\begin{tabular}{lcc}
\hline Actividades económicas & $\begin{array}{c}\text { Horas de trabajo } \\
\text { (variación en \%) }\end{array}$ & Empleo (variación en \%) \\
\hline Minería y actividades extractivas & 1,25 & $-4,24$ \\
Manufacturas & 1,37 & $-14,51$ \\
Suministro de electricidad y gas & 1,88 & 5,36 \\
Suministro de agua & 1,75 & $-11,11$ \\
Construcción & 2,07 & $-22,20$ \\
Comercio al por mayor y menor & 0,92 & $-9,15$ \\
Transporte y almacenamiento & 0,93 & $-7,16$ \\
Hostelería y restauración & 1,22 & 0,50 \\
Información y comunicación & $-0,26$ & $-10,42$ \\
Finanzas y seguros & 2,12 & $-0,49$ \\
Actividades inmobiliarias & 0,45 & $-25,22$ \\
Actividades profesionales, científicas y técnicas & 0,40 & $-1,71$ \\
Actividades administrativas & 0,13 & $-3,51$ \\
Administración pública y defensa & 0,65 & 5,62 \\
Educación & 1,53 & 3,68 \\
Salud y trabajo social & 1,93 & 1,53 \\
Otros servicios & $-1,24$ & $-2,85$ \\
\hline
\end{tabular}

Fuente: elaboración propia con datos del Instituto Nacional de Estadística (INE).

Según Woo (2012), "si hay un problema grave de desempleo estructural, entonces los sectores en los que tienen problemas para encontrar trabajadores deberían subir los salarios" (p. 5). Luego, al observar la evolución de los costos salariales totales y el tiempo extralaboral (ver Figura $\underline{3}$ ), en el período de ejecución del plan de estímulo, se destaca el aumento de los costos laborales en los sectores identificado para impulsar la demanda laboral durante este período. 
Hospedaje y hostelería

Actividades técnicas, profesionales y científicas

Finanzas y seguros

Arte, entretenimiento y actividades recreativas

Manufacturas

Servicios administrativos y de apoyo

Información y comunicación

Otros servicios

Comercio al por mayor y al detall

Servicios inmobiliarios

Transporte y almacenamiento

Constucción

Suministro de Agua

Educación

Minería e industrias extractivas

Administraciones Públicas y defensa

Salud y trabajo social

Electricidad, gas, vapor y suministro de aire...
$-4,71$

\section{$-3,51$}

$-3,32$

$-37,8$

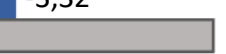

$-35,25$

$-6,04$

$-31,54$

$-3,53$

$-5,61$

$-31,03$

$-26,17$

$-2,49$

$-24,51$

1,66

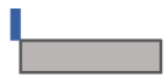

$-22,71$

2,94

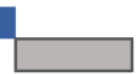

$-18,84$

$-1,99$

$-13,64$

0,08

$-2,51$

1,12 $-1,49$

0,99

1,23

4,92

7,03

$$
12,27
$$$$
9,04
$$

20,28

5,37

26,52

7,29

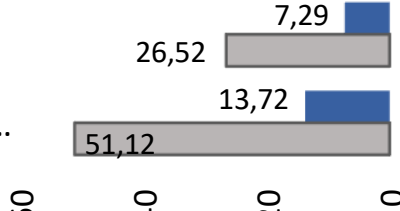

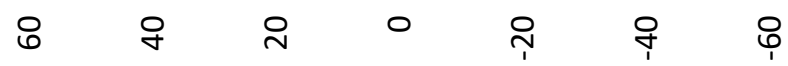

चariación salarios reales $\quad \square$ Variación ingresos por horas extraordinarias

Figura 3. Variación de los salarios reales y de los ingresos por horas extras, por actividades económicas, durante el período comprendido entre el IV trimestre de 2007 y el II de 2010. Fuente: elaboración propia con datos del INE 


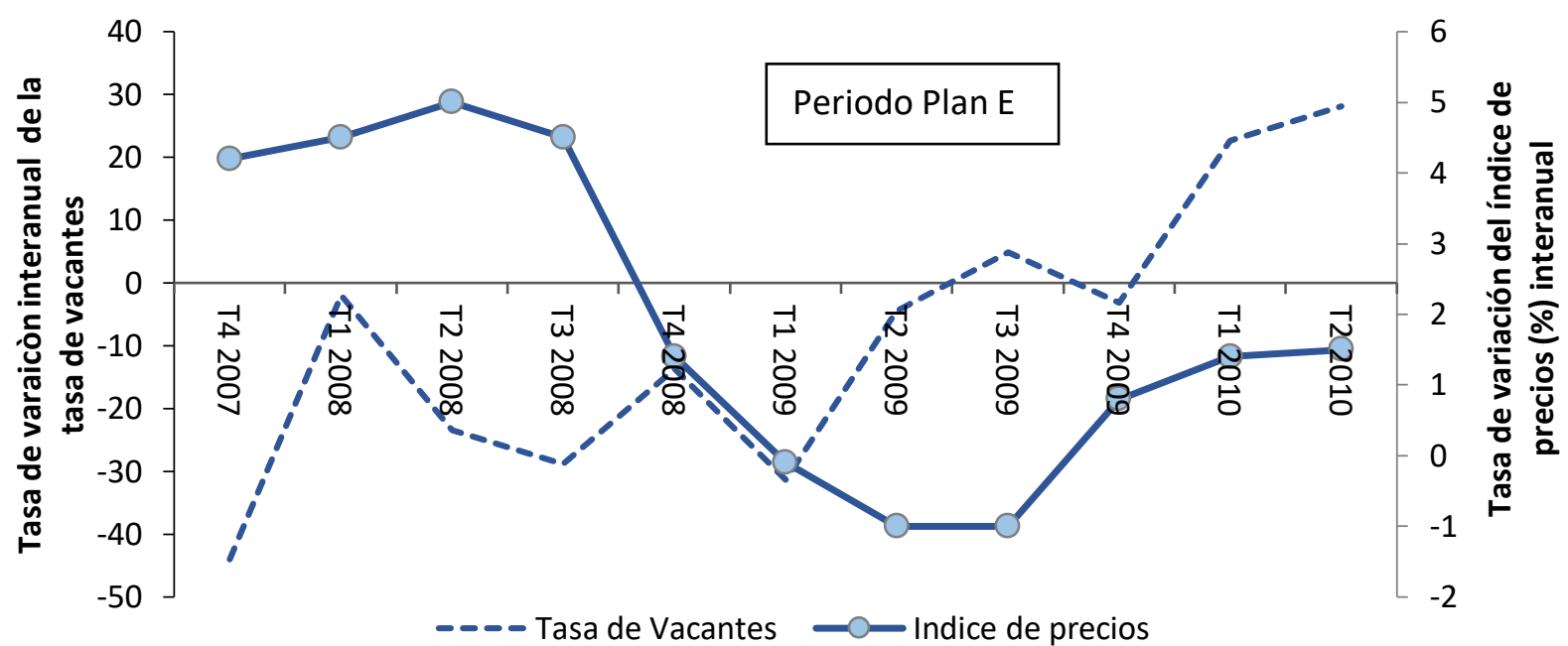

Figura 4. Crecimiento interanual de las vacantes de empleo y del índice de precios, durante el período comprendido entre el IV trimestre de 2007 y el II de 2010. Elaboración propia a partir de datos del Ministerio de Trabajo y SS y del Instituto Nacional de estadísticas, ambos de España.

Este aumento, experimentado en los costos laborales en las industrias que ocupaban el 2,9 por ciento del empleo total en el segundo trimestre de 2010, podría haber tenido un impacto negativo en el equilibrio macroeconómico, principalmente en el aumento del nivel de precios. En este sentido, hay que considerar lo siguiente:

Si el desempleo es 'estructural' entonces la política gubernamental que busca aumentar la demanda -por ejemplo, bajas tasas de interés o estímulo fiscal (como el Plan E) - tendrá poco o ningún efecto en la tasa de desempleo nacional e incluso podría empeorar las cosas al encender la inflación. (Woo, 2012 , p. 1) $)^{12}$

Desde el comienzo del plan de estímulo, es importante tener en cuenta el proceso de crecimiento experimentado por las vacantes de empleo. Este crecimiento también está acompañado por una evolución positiva de la tasa de inflación, que parece estar rezagada entre dos y tres trimestres relacionados con la evolución de las vacantes, comenzando la fase de crecimiento de la inflación desde el tercer trimestre de 2009 (ver Figura 4).

Esta evolución procíclica y rezagada del nivel de precios en la evolución de la desocupación se debe al tiempo que tardan los agentes económicos en actualizar sus expectativas e incorporarlas al proceso de negociación de las condiciones laborales, principalmente a través de la negociación colectiva. En este sentido, el aumento simultáneo del nivel de vacantes en diferentes industrias, que indica un empeoramiento del proceso de desajuste en el mercado laboral, hace que las empresas necesiten utilizar intensamente a su personal para satisfacer el aumento en la demanda de bienes y servicios. servicios en el mercado. Esto implica un crecimiento en el número de horas de trabajo para el personal de la empresa, porque no es posible encontrar quien trabaje con las mismas habilidades en el mercado. Esto aumenta el

\footnotetext{
12 Véase también Sell \& Reinisch (2013)
} 
poder del personal para exigir salarios más altos en el proceso de negociación colectiva, si el aumento de la producción continúa en un mediano plazo (Layard, Nickel \& Jackman, 1994). Por lo tanto, este proceso produciría un impacto negativo en los costos de producción y el nivel de inflación.

\section{Un análisis empírico: El caso español}

En este estudio, realizamos tres análisis diferentes, cada uno para BC, PC y MOG, para el período comprendido entre el cuarto trimestre de 2007 y el segundo trimestre de 2010 . Eso es durante el comienzo de la crisis económica -y de empeoramiento del desajuste del mercado laboral en España (Ruesga, Silva \& Pérez, 2015)- y el plan económico de estímulo llevado a cabo por el gobierno en curso. El objetivo de esta sección es demostrar la relevancia empírica del MOG, utilizando técnicas econométricas, para explicar tal resultado.

Para el análisis de la BC y su estabilidad consideramos la metodología propuesta por Wall \& Zoega (2002), Bouvet (2011), Sell \& Rheinisch (2013), Dixon, Lim \& Freebairn (2014) y Ruesga, Silva \& Pérez (2015), estimando la siguiente ecuación:

$$
\operatorname{Ln} u_{i t}=\beta_{0}+\beta_{1} \cdot \operatorname{Ln} v_{i t}+\beta_{2} \cdot \operatorname{Ln} v_{i t}^{2}+\lambda_{t}+\varepsilon_{i t}(1)
$$

Siendo $u_{i t}$ la tasa de desempleo y $v_{i t}$ la tasa de vacantes, ambas expresadas en registros para la región "i" en el trimestre " $t$ "13, y $\lambda_{t}$ los efectos de tiempo. En esta ecuación, incluimos el cuadrado de $v_{\text {it }}$ para probar la convexidad de esta función. Los coeficientes estimados para los efectos de tiempo determinarán la estabilidad de la BC a lo largo del período analizado.

Por otro lado, para las estimaciones de PC utilizamos la metodología utilizada por Sell \& Rheinisch. (2013):

$$
\pi_{i t}=\beta_{0}+\beta_{1} \cdot u_{i t}+\beta_{2} \cdot u_{i t}^{2}+\lambda_{\text {year }}+\varepsilon_{i t}(2)
$$

Aquí la tasa de inflación $\left(\pi_{\mathrm{it}}\right)$ depende de la tasa de desempleo $\left(u_{i t}\right)$, su cuadrado y de los efectos de tiempo para cada año $\left(\lambda_{t}\right)$.

Finalmente, estimamos el MOG utilizando la misma metodología propuesta por Sell \& Rheinisch (2013), incluyendo nuevamente el cuadrado de la variable dependiente:

$$
\pi_{i t}=\beta_{0}+\beta_{1} \cdot v_{i t}+\beta_{2} \cdot v_{i t}^{2}+\lambda_{\text {year }}+\varepsilon_{i t}(3)
$$

Debido a que el posible empeoramiento del proceso de desajuste en el mercado laboral puede no ocurrir de forma inmediata con el aumento de los costos laborales, estimamos la ecuación (3) para diferentes rezagos de la tasa de vacantes, alternando en la regresión la variable en el

\footnotetext{
${ }^{13}$ La forma funcional comúnmente utilizada para representar la CB es la de una función Cobb-Douglas (Wall y Zoega, 2002): $M=m(U, V)=A \cdot U^{\alpha} \cdot V^{1-\alpha}$, donde el número de contrataciones (M) es dependiente del número de ajustes en el mercado laboral $(m(\cdot))$ entre desempleo $(U)$ y puestos vacantes $(V)$. Es necesario llevar a cabo la linearización de esta ecuación aplicando la transformación logarítmica para sus estimaciones. 86
}

Santos M. Ruesga, Laura Pérez Ortiz y Friedrich L. Sell 
período 't-2', 't-3' y 't-4'14. Se utilizan tres rezagos diferentes en la tasa de vacantes por su correlación significativa con el nivel de precios (Tablas 2 y 3 , para los rezagos ' $\mathrm{t}-2$ ' y ' $\mathrm{t}$-4') que muestra la correlación entre ambas variables con diferentes rezagos en la tasa de vacantes). Esto demuestra el efecto rezagado de la tasa de vacantes en la inflación ${ }^{15}$.

Tabla 2

Estimación de la MOG utilizando dos retrasos para la tasa de vacantes (Vacancies it)

\begin{tabular}{|c|c|c|c|c|c|c|}
\hline & $\begin{array}{l}\text { FE-OLS } \\
\text { (1) }\end{array}$ & $\begin{array}{l}\text { RE-GLS } \\
\text { (2) }\end{array}$ & $\begin{array}{l}\text { FE-OLS } \\
\text { (3) }\end{array}$ & $\begin{array}{l}\text { RE-GLS } \\
\text { (4) }\end{array}$ & $\begin{array}{l}\text { Sistema } \\
\text { GMM (5) }\end{array}$ & $\begin{array}{l}\text { Sistema } \\
\text { GMM (6) }\end{array}$ \\
\hline$\gamma_{2008}$ & $\begin{array}{r}-0.938 * * * \\
(0.051)\end{array}$ & $\begin{array}{r}-0.952^{* * *} \\
(0.045)\end{array}$ & $\begin{array}{r}-0.922^{* * *} \\
(0.059)\end{array}$ & $\begin{array}{r}-0.939 * * * \\
(0.051)\end{array}$ & $\begin{array}{r}-1.249 * * * \\
(0.061)\end{array}$ & $\begin{array}{r}-1.070^{* * *} \\
(0.084)\end{array}$ \\
\hline$\gamma_{2009}$ & $\begin{array}{r}-1.480 * * * \\
(0.074)\end{array}$ & $\begin{array}{r}-1.502^{* * *} \\
(0.057)\end{array}$ & $\begin{array}{r}-1.458 * * * \\
(0.084)\end{array}$ & $\begin{array}{r}-1.485^{* * *} \\
(0.063)\end{array}$ & $\begin{array}{r}0.395^{* * *} \\
(0.102)\end{array}$ & $\begin{array}{r}0.537 * * * \\
(0.182)\end{array}$ \\
\hline$\gamma_{2010}$ & $\begin{array}{r}-1.121 * * * \\
(0.055)\end{array}$ & $\begin{array}{r}-1.135^{* * *} \\
(0.042)\end{array}$ & $\begin{array}{r}-1.092^{* * *} \\
(0.062)\end{array}$ & $\begin{array}{r}-1.112^{* * *} \\
(0.048)\end{array}$ & $\begin{array}{r}-0.124^{* *} \\
(0.081)\end{array}$ & $\begin{array}{r}0.033 \\
(0.139)\end{array}$ \\
\hline Vacancies $_{i t-2}$ & $\begin{array}{r}0.575 \\
(0.352)\end{array}$ & $\begin{array}{l}0.400 * \\
(0.222)\end{array}$ & $\begin{array}{r}1.243 \\
(0.950)\end{array}$ & $\begin{array}{r}0.974 \\
(0.703)\end{array}$ & $\begin{array}{r}1.003 * * * \\
(0.340)\end{array}$ & $\begin{array}{l}5.979 * \\
(3.176)\end{array}$ \\
\hline Vacancies $_{i t-2}^{2}$ & - & - & $\begin{array}{l}-0.688 \\
(0.697)\end{array}$ & $\begin{array}{l}-0.605 \\
(0.557)\end{array}$ & - & $\begin{array}{l}-4.860 \\
(3.475)\end{array}$ \\
\hline Inflation $_{i t-1}$ & - & - & - & - & $\begin{array}{r}1.402^{* * *} \\
(0.067)\end{array}$ & $\begin{array}{r}1.336^{* * *} \\
(0.118)\end{array}$ \\
\hline Inflation $_{\text {it }-2}$ & - & - & - & - & $\begin{array}{r}-0.117^{* * *} \\
(0.021)\end{array}$ & $\begin{array}{r}-0.062^{*} \\
(0.035)\end{array}$ \\
\hline Inflation $_{i t-3}$ & - & - & - & - & $\begin{array}{r}0.299 * * * \\
(0.030)\end{array}$ & $\begin{array}{r}0.228 * * * \\
(0.042)\end{array}$ \\
\hline$\beta_{0}$ & $\begin{array}{r}1.300 * * * \\
(0.159)\end{array}$ & $\begin{array}{r}1.375^{* * *} \\
(0.102)\end{array}$ & $\begin{array}{r}1.157^{* * * *} \\
(0.273)\end{array}$ & $\begin{array}{r}1.256 * * * \\
(0.195)\end{array}$ & $\begin{array}{r}-0.328^{* *} \\
(0.159)\end{array}$ & $\begin{array}{r}-1.382 * * \\
(0.662)\end{array}$ \\
\hline Número de grupos & 17 & 17 & 17 & 17 & 17 & 17 \\
\hline $\begin{array}{l}H_{0}: \gamma_{2008}=\gamma_{2009}= \\
\gamma_{2010}=0^{\wedge}\end{array}$ & 0.000 & 0.000 & 0.000 & 0.000 & 0.000 & 0.000 \\
\hline$\frac{\text { dInflatıo }_{l t}}{\text { dVacancies }_{i t}}$ & - & - & $\begin{array}{r}0.771 \\
(0.507)\end{array}$ & $\begin{array}{l}0.559 * \\
(0.339)\end{array}$ & - & $\begin{array}{r}2.643^{* * *} \\
(0.875)\end{array}$ \\
\hline Test Mundlack ${ }^{\wedge 16}$ & & 0.3563 & & 0.3081 & - & - \\
\hline Test Hansen^17 & - & - & - & - & 0.917 & 0.902 \\
\hline $\begin{array}{l}\text { Test Arellano-Bond }{ }^{18} \\
\operatorname{AR}(1)^{\wedge}\end{array}$ & - & - & - & - & 0.002 & 0.004 \\
\hline Test Arellano-Bondt $\mathrm{AR}(2)^{\wedge}$ & - & - & - & - & 0.937 & 0.445 \\
\hline
\end{tabular}

^p-valor. *significativo al $10 \%,{ }^{* *}$ significativo al $5 \%, y * *$ significativo al $1 \%$. En las estimaciones con GMM, se utilizan como instrumentos los segundo y tercer retardos y la primera diferencia de la variable endógena $\left(\log\right.$ Inflation $\left._{i t}\right)$. Fuente: elaboración propia.

\footnotetext{
14 También se estima la ecuación (3) para la tasa de vacantes en 't' y 't-1'. Los resultados indican una relación negativa y significativa entre la tasa de vacantes y el nivel de precios para 't' y una relación positiva y significativa (como se esperaba) para 't-1'. Los resultados están disponibles para quienes los soliciten al equipo autor de este artículo.

15 Por razones de espacio no se ha incluido la tabla correspondiente al rezago ' $t$-3', en la que se observan resultados similares a las de las dos incorporadas al texto. Asimismo, como se señala en la nota anterior, los resultados están disponibles.

${ }^{16}$ Véase Mundlak (1978).

${ }^{17}$ Véase Hansen (2001)

${ }^{18}$ Véase Arellano \& Bond (1991).
} 
Los datos utilizados en el análisis son observaciones para 17 regiones españolas ${ }^{19}$, por un período que va desde el cuarto trimestre de 2007 hasta el segundo de 2010, lo cual permite utilizarlos como un conjunto de datos del pane ${ }^{20}$. Como se ha señalado ya, los datos provienen de dos fuentes diferentes: el número de asalariados ocupados en el mercado laboral, de la Encuesta de Población Activa (EPA), y las vacantes ${ }^{21}$ disponibles en el mercado laboral, de la Encuesta Trimestral de Coyuntura Laboral (ETCL).

Tabla 3

Estimación de la MOG utilizando cuatro retrasos para la tasa de vacantes (Vacancies it)

\begin{tabular}{|c|c|c|c|c|c|c|}
\hline & $\begin{array}{r}\text { FE-OLS } \\
(1)\end{array}$ & $\begin{array}{r}\text { RE-GLS } \\
(2)\end{array}$ & $\begin{array}{r}\text { FE-OLS } \\
(3)\end{array}$ & $\begin{array}{r}\text { RE-GLS } \\
(4)\end{array}$ & $\begin{array}{r}\text { Sistema } \\
\text { GMM (5) }\end{array}$ & $\begin{array}{r}\text { Sistema } \\
\mathrm{GMM}(6)\end{array}$ \\
\hline$\gamma_{2008}$ & $\begin{array}{r}-0.953^{* * *} \\
(0.040)\end{array}$ & $\begin{array}{r}-0.965^{* * *} \\
(0.038)\end{array}$ & $\begin{array}{r}-0.951^{* * *} \\
(0.040)\end{array}$ & $\begin{array}{r}-0.957 * * * \\
(0.041)\end{array}$ & $\begin{array}{r}-1.165^{* * *} \\
(0.077)\end{array}$ & $\begin{array}{r}-1.148^{* * *} \\
(0.084)\end{array}$ \\
\hline$\gamma_{2009}$ & $\begin{array}{r}-1.474 * * * \\
(0.058)\end{array}$ & $\begin{array}{r}-1.504 * * * \\
(0.046)\end{array}$ & $\begin{array}{r}-1.471 * * * \\
(0.059)\end{array}$ & $\begin{array}{r}-1.493 * * * \\
(0.050)\end{array}$ & $\begin{array}{r}0.286 * * * \\
(0.094)\end{array}$ & $\begin{array}{r}0.285 * * * \\
(0.101)\end{array}$ \\
\hline$\gamma_{2010}$ & $\begin{array}{r}-1.074 * * * \\
(0.060)\end{array}$ & $\begin{array}{r}-1.110^{* * *} \\
(0.041)\end{array}$ & $\begin{array}{r}-1.071^{* * *} \\
(0.062)\end{array}$ & $\begin{array}{r}-1.098 * * * \\
(0.048)\end{array}$ & $\begin{array}{l}-0.017 \\
(0.074)\end{array}$ & $\begin{array}{r}-0.003 \\
(0.103)\end{array}$ \\
\hline Vacancies $_{i t-4}$ & $\begin{array}{r}0.532 \\
(0.309)\end{array}$ & $\begin{array}{l}0.331^{*} \\
(0.195)\end{array}$ & $\begin{array}{r}0.718 \\
(0.916)\end{array}$ & $\begin{array}{r}0.748 \\
(0.773)\end{array}$ & $\begin{array}{r}1.792^{* * *} \\
(0.429)\end{array}$ & $\begin{array}{r}2.308^{* *} \\
(0.941)\end{array}$ \\
\hline Vacancies $_{\text {it }-4}^{2}$ & - & - & $\begin{array}{r}-0.224 \\
(1.020)\end{array}$ & $\begin{array}{r}-0.461 \\
(0.811)\end{array}$ & - & $\begin{array}{r}-0.593 \\
(1.060)\end{array}$ \\
\hline Inflation $_{i t-1}$ & - & - & - & - & $\begin{array}{r}1.308^{* * *} \\
(0.089)\end{array}$ & $\begin{array}{r}1.302^{* * *} \\
(0.096)\end{array}$ \\
\hline Inflation $_{i t-2}$ & - & - & - & - & $\begin{array}{r}-0.229 * * * \\
(0.021)\end{array}$ & $\begin{array}{r}-0.238 * * * \\
(0.023)\end{array}$ \\
\hline Inflation $_{i t-3}$ & - & - & - & - & $\begin{array}{r}0.278^{* * *} \\
(0.040)\end{array}$ & $\begin{array}{r}0.278^{* * *} \\
(0.049)\end{array}$ \\
\hline$\beta_{0}$ & $\begin{array}{r}1.298 * * * \\
(0.143) \\
\end{array}$ & $\begin{array}{r}1.393^{* * *} \\
(0.086) \\
\end{array}$ & $\begin{array}{r}1.265^{* * *} \\
(0.209) \\
\end{array}$ & $\begin{array}{r}1.309 * * * \\
(0.177)\end{array}$ & $\begin{array}{r}-0.534 * * * \\
(0.162) \\
\end{array}$ & $\begin{array}{r}-0.629 * * * \\
(0.224) \\
\end{array}$ \\
\hline Número de grupos & 17 & 17 & 17 & 17 & 17 & 17 \\
\hline $\begin{array}{l}H_{0}: \gamma_{2008}=\gamma_{2009}=\gamma_{2010}= \\
0^{\wedge}\end{array}$ & 0.000 & 0.000 & 0.000 & 0.000 & 0.000 & 0.000 \\
\hline 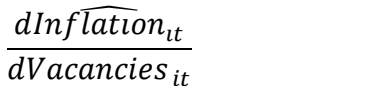 & - & - & $\begin{array}{l}0.555^{*} \\
(0.327)\end{array}$ & $\begin{array}{l}0.412^{*} \\
(0.239)\end{array}$ & - & $\begin{array}{r}1.875^{* * *} \\
(0.450)\end{array}$ \\
\hline Test Mundlack^ & & 0.5333 & & 0.5648 & - & - \\
\hline Test Hansen^^ & - & - & - & - & 0.923 & 0.897 \\
\hline Test Arellano-Bond $A R(1)^{\wedge}$ & - & - & - & - & 0.001 & 0.001 \\
\hline Test Arellano-Bond AR(2)^ & - & - & - & - & 0.503 & 0.637 \\
\hline
\end{tabular}

${ }^{\wedge} \mathrm{p}$-valor. ${ }^{*}$ significativo al $10 \%,{ }^{* *}$ significativo al $5 \%, y * *$ significativo al $1 \%$. En las estimaciones con GMM, se utilizan como instrumentos los segundo y tercer retardos y la primera diferencia de la variable endógena $\left(\log\right.$ Inflation $\left._{i t}\right)$. Fuente: elaboración propia.

\footnotetext{
${ }^{19}$ No se consideran para el análisis los datos relativos a las ciudades autónomas de Ceuta y de Melilla a causa de su reducida dimensión.

${ }^{20}$ En lugar de utilizar datos de series temporales, existe un tipo de análisis de datos de panel que podría ser más adecuado, pues permite controlar la heterogeneidad observable y no observable, desde la lógica de que cada región que comprende el mercado funciona de manera diferente, evitando la posible existencia de sesgo de agregación que afecta las estimaciones (véase Börsch-Supan, 1991).

${ }^{21}$ Esta fuente define vacante de empleo como el puesto de trabajo no ocupado que existe el último día del trimestre de referencia, en el momento de la elaboración de la muestra. El ente empleador podrá ocupar esta vacante con un trabajador proveniente del propio personal de la empresa o acudiendo al mercado laboral externo.
}

88

Santos M. Ruesga, Laura Pérez Ortiz y Friedrich L. Sell 
En el análisis, se elaboran tres modelos de datos de panel diferentes: de efectos fijos (FE), de efectos aleatorios (RE) y un sistema GMM (método generalizado de momentos) (Arellano \& Bover, 1995; Blundell \& Bond, 1998), el ultimo se aplica para obtener estimadores consistentes y eficientes para las ecuaciones estimadas, incluidos rezagos de las variables dependientes.

Las estimaciones ${ }^{22}$ para la $\mathrm{BC}$ indican que el modelo del sistema GMM, el último para obtener estimadores es el más adecuado para el análisis. Esta estimación muestra una elasticidad negativa entre el desempleo y la tasa de vacantes de -0.06 puntos. Los resultados de interés son los coeficientes de tiempo estimados $\left(\lambda_{\text {year }}\right)$, que indican un cambio positivo y significativo de la curva, especialmente intenso durante los años 2008 y 2009, con un coeficiente estimado de 0.109 y 0.127 para cada uno, respectivamente, donde la curva experimenta un movimiento positivo con respecto a 2007. Estos resultados indican un peor comportamiento de la eficiencia del mercado laboral durante la recesión.

Estos resultados son similares a los obtenidos por Haincourt \& Mogliani (2012), y Ruesga, Silva \& Pérez (2015), quienes concluyen que la menor eficiencia en el ajuste del mercado laboral se debe al cambio en la estructura sectorial del empleo relacionado con el estallido de la burbuja inmobiliaria y la caída del sector de la construcción. Así, citando a Bouvet (2011, p.9), "los cambios sectoriales inducen el desajuste de habilidades entre los trabajadores desempleados y los puestos de trabajo no cubiertos, lo que lleva a cambiar la BC hacia afuera", algo que ha sucedido en España durante la Gran Recesión.

En cuanto a las estimaciones de la PC, similares a las de la BC, el modelo que mejor se ajusta es el método generalizado de momentos GMM. En este modelo, para la PC, los coeficientes muestran una relación negativa y significativa esperada entre la inflación y la tasa de desempleo de -8.38 puntos. Además, los coeficientes de los efectos de tiempo indican un desplazamiento hacia afuera de esta curva para los años 2009 y 2010, similar a las estimaciones obtenidas para la BC. Aquí, la menor eficiencia en el proceso de correspondencia y el efecto del programa económico han afectado el equilibrio de las PC, aumentando el nivel de desempleo, lo que resulta en un cambio positivo de esta curva y su equilibrio en el largo plazo (Sell \& Rheinisch, 2013). Sin embargo, este camino de crecimiento en el cambio de la PC hacia el exterior parece desacelerarse en 2009. Podría ser una consecuencia del efecto del Plan E, que favoreció la desaceleración en el crecimiento de la tasa de desempleo desde el comienzo del año.

Considerando las estimaciones del MOG -aquí se incluyen las estimaciones de GMM sin la variable dependiente cuadrada (Vacancies ${ }_{i t-n}^{2}$ ) en todas las regresiones aplicadas, los resultados indican un efecto positivo y significativo entre la tasa de desocupación y la tasa de inflación, cuando la primera se retrasa en dos o cuatro trimestres ${ }^{23}$ (Tablas $\underline{2}$ y $\underline{3}$ ). Al analizar

\footnotetext{
${ }^{22}$ Todas las variables utilizadas en las diferentes estimaciones se han ajustado estacionalmente (ver Fok, Franses \& Paap, 2005).

${ }^{23}$ El coeficiente estimado entre la tasa de vacantes de empleo y la inflación se mueve entre 0.5 y 1.79 puntos porcentuales.
} 
la dinámica del MOG con los resultados obtenidos para las variables de tiempo ( $\lambda_{\text {year }}$ ), encontramos un cambio significativo de la curva, hacia el interior del eje de coordenadas, en $2008^{24}$. La curva toma el signo opuesto a partir de 2009 , con un coeficiente estimado para $\lambda_{2009}$ que es significativo: pasa de 0,286 a 0,403, lo que indica que el MOG se mueve hacia afuera con respecto a 2007. Este cambio positivo coincide con el peor comportamiento de la eficiencia del mercado laboral en este período, similar al que se muestra en la Figura $\underline{1}$ e influye en el movimiento del MOG hacia afuera.

No obstante, durante 2010 se observa que el MOG sufre un cambio significativo del signo opuesto, hacia adentro, lo que indica una peor relación entre el nivel de precios y las vacantes de mercado en relación con la situación al comienzo de la crisis. Este resultado proporciona evidencia empírica de que el plan de estímulo del gobierno implementado en un momento de pérdida de eficiencia en el mercado laboral habría afectado negativamente el nivel de precios, causando un empeoramiento en la ratio inflación/vacantes. Vale la pena resaltar que el empeoramiento de la relación se retrasó, ya que el plan de estímulo comenzó en noviembre de 2008 y su efecto sobre la estabilidad del MOG comenzó a notarse en 2010, según nuestras estimaciones. Este retraso estaría asociado con el tiempo que lleva la transformación de una mayor demanda de empleo no cubierto en el mercado en costos salariales, y estos en precios de mercado.

Finalmente, la Figura 5 muestra la dinámica de la MOG estimada, teniendo en cuenta los diferentes movimientos identificados por la BC y la PC a lo largo del período de tiempo analizado. Por lo tanto, se han de considerar dos fases diferentes que afectan a la MOG:

- La primera, en la que se observa un peor ajuste en el mercado laboral, con el consiguiente impacto en la estabilidad de la BC, combinado con una fuerte caída en la demanda agregada

- y la segunda, con el plan de estímulo económico iniciado por el gobierno para aumentar el nivel de empleo y su impacto en la PC.

El equilibrio inicial en la Figura $\underline{5}$ se ubica en el punto a del cuadrante I, con un nivel de desempleo $\left(u_{0}\right)$ y un nivel de vacantes $\left(v_{0}\right)$ definido en la $B C_{0}$. El equilibrio se altera por la recesión y el colapso del sector de la construcción en España. Este proceso produce un desplazamiento hacia afuera de la $\mathrm{BC}$, que se mueve hacia un punto de equilibrio situado en $b$, con un mayor desempleo $\left(u_{1}\right)$ y niveles de vacantes más bajos $\left(v_{1}\right)$, como es habitual en un

\footnotetext{
${ }^{24}$ Este resultado, inicialmente sería inesperado de acuerdo con la dinámica del MOG y puede deberse al impacto en los precios del alza en el precio del crudo en 2008, que alcanzó una cotización sin precedentes a comienzos de julio de ese año. Este rápido crecimiento de los precios -con una variación interanual promedio de 4,1 por ciento, 1,3 puntos porcentuales por encima de la media de 2007 (datos del Instituto Nacional de Estadística Español), como resultado del shock externo sufrido- podría haber dado lugar al nivel más alto de la relación inflación-vacantes, y provocando que la curva del MOG se mueva hacia adentro. Sin embargo, una vez estabilizado el precio del petróleo, el MOG tenderá a permanecer sin cambios o, si se corrige el choque externo, volverá a su posición inicial.
}

90

Santos M. Ruesga, Laura Pérez Ortiz y Friedrich L. Sell 
período de recesión combinado con desajustes en el mercado laboral (ver Hobijn \& Sahin, 2012).

El impacto negativo de la recesión en la demanda agregada hace que el aumento del desempleo y la menor demanda en el mercado laboral depriman el nivel de precios en el corto plazo, desplazando el equilibrio de las PC al punto b' (véase el cuadrante II). El nuevo equilibrio en la BC y la PC altera la estabilidad del MOG (en el corto plazo ${ }^{25}$ ), desplazándolo hacia afuera (con el equilibrio a" en $\mathrm{MOG}_{0}$ moviéndose a $\mathrm{b}^{\prime \prime}$ en $\mathrm{MOG}_{1}$ ), donde los niveles de inflación y vacantes son inferiores.

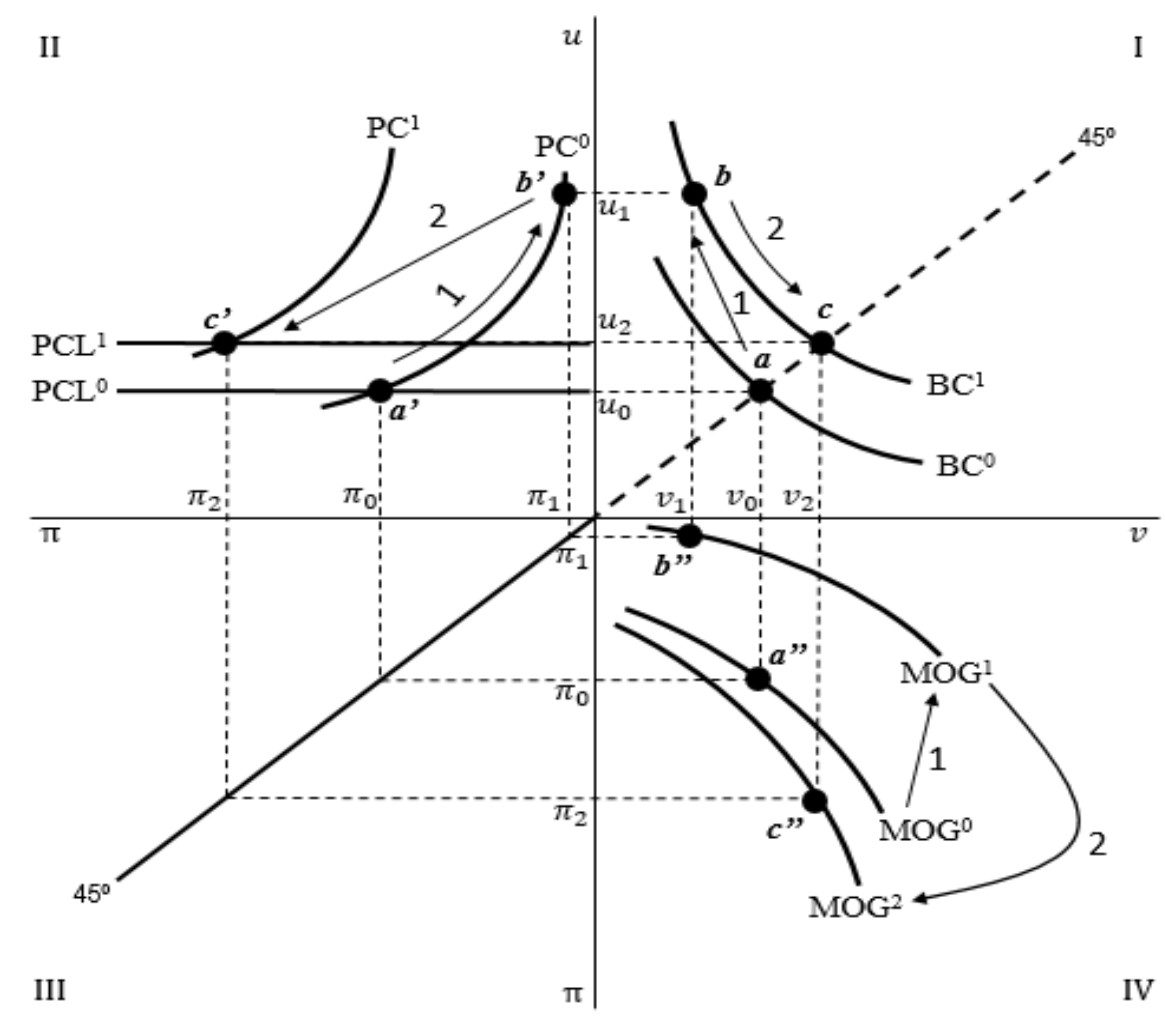

Figura 5. Interpretación de la dinámica de la curva MOG según las estimaciones realizadas Fuente: elaboración propia.

Esta descripción se refiere a la primera fase de la dinámica de MOG, en la que empeora la correspondencia entre oferta y demanda en el mercado laboral; las flechas 1 identifican estos movimientos en la Figura 5.

En la segunda fase, identificada con las flechas 2 en la misma figura, el efecto de un plan de estímulo económico sobre el equilibrio del MOG es el siguiente. Por un lado, el plan de estímulo afecta positivamente el nivel de precios en el corto plazo, motivado por el impacto de la demanda agregada y el cambio de los agentes económicos en las expectativas de precios,

${ }^{25}$ Dado que se está examinando el corto plazo (el análisis se realiza solo para el período comprendido entre el cuarto trimestre de 2007 y el segundo de 2010), no se considera el efecto que un cambio externo de la BC podría tener en la PC a largo plazo, como se contempla en la Figura 1 
desplazando las $\mathrm{PC}$ hacia afuera (de $\mathrm{PC}_{0}$ a $\mathrm{PC}_{1}$ ). Como resultado, el equilibrio en el cuadrante II de la Figura 5 cambia de b' a c', donde el desempleo es más bajo y el nivel de precio es más alto. El impacto de la demanda agregada ofrece a las empresas mayores incentivos para contratar en el mercado laboral, aumentando el número de vacantes existentes y disminuyendo el desempleo (situándolo en $u_{2}$ y $v_{2}$, respectivamente) y colocando el equilibrio en el cuadrante I en el punto c. El desplazamiento hacia afuera ocurrido en la PC tiende a provocar que la función MOG retroceda hacia adentro, como se describe en la dinámica de la Figura 2, moviendo el equilibrio de b" en $\mathrm{MOG}_{1}$ a $\mathrm{c}^{\prime \prime}$ en $\mathrm{MOG}_{2}$, donde la inflación y los niveles de vacantes tienden a ser menos favorables.

En general, los resultados confirman que el empeoramiento del proceso de desajuste experimentado en el mercado laboral español combinado con la aplicación del Plan E, afectó negativamente al equilibrio de la MOG durante la crisis económica. Por tanto, se puede contemplar, al mismo tiempo, un mayor nivel de vacantes y una tasa de inflación más elevada, debido a la falta de asalariados cualificados para ocupar las vacantes generadas por las empresas y, en consecuencia, un uso intensivo de la plantilla contratada. Todo lo cual genera una mayor presión sobre los costos laborales, ya que los grupos trabajadores demandan salarios más altos en la negociación colectiva. En consecuencia, los costos laborales aumentan para la empresa y presionan hacia la inflación y al alza.

\section{Conclusiones}

Al combinar los conceptos, de la BC y la PC, se genera una relación explícita entre la tasa de inflación y la tasa de vacantes, que se ha etiquetado como MOG. Dado el razonamiento teórico sobre esta relación aquí explicado, la correlación empírica entre estas dos variables macroeconómicas debería ser positiva. De manera concisa, la idea es que la tasa de vacantes observada puede ser una señal de un desequilibrio significativo o un grado de escasez de habilidades en el mercado laboral. Si hay una carencia de mano de obra cualificada en el mercado junto con un aumento de la ratio de vacantes, los salarios aumentarán debido a un mayor poder de negociación de los empleados calificados para negociar sus condiciones laborales. Los empleadores consideran este efecto del precio de los factores y recalculan los precios de los bienes, lo que, ceteris paribus, dará como resultado una tasa de inflación observada más alta.

En esta investigación, se ha analizado el caso español, tomando en consideración el período comprendido entre el comienzo de la recesión económica (2007) y el final del plan de estímulo para la economía (2010), introducido por el gobierno español con el fin de impulsar el crecimiento económico. Este país y el período son ideales para analizar la consistencia empírica del MOG. Las evidencias obtenidas indican que el mercado laboral español se ha enfrentado a un proceso de desajuste cada vez mayor que limita la adaptación del mercado de trabajo a la creciente demanda en el mercado de bienes y servicios, cuando esta última estaba impulsada por el plan de estímulo económico.

El colapso del modelo económico español antes de la crisis económica, gobernada por el sector de la construcción, afectó las industrias que fueron pioneras en el crecimiento 92 
económico y del empleo durante 1995-2007. Estas industrias ocuparon personal con un nivel de educación bajo a medio bajo, lo que afectó negativamente su posibilidad de encontrar un nuevo trabajo durante la recesión.

En conclusión, este análisis enfatiza la necesidad de combinar simultáneamente políticas públicas de estímulo económico y reformas estructurales para expandir la demanda agregada y adaptar la economía a un nuevo modelo económico. Si no fuera así, como mostramos en este artículo, los efectos derivados de solo una política fiscal expansiva pueden ser rápidamente neutralizados por un proceso inflacionario sustentando en la situación del mercado laboral, caracterizado por un gap ocupacional importante, que impulsa el alza de los salarios en plena recesión.

Según el presidente del Banco de la Reserva Federal de Filadelfia, Charles Plosser (O'Grady 2011, p. 1):

"You can't change the carpenter into a nurse easily, and you can't change the mortgage broker into a computer expert in a manufacturing plant very easily. Eventually that stuff will work itself out. People will be retrained and they'll find jobs in other industries. But monetary (and fiscal) policy can't retrain people. Monetary (and fiscal) policy can't fix those problems". [No se puede convertir al carpintero en una enfermera fácilmente, y no se puede convertir fácilmente al corredor de hipotecas en un experto en informática en una industria manufacturera. Eventualmente, esas cosas funcionarán por sí solas. Las personas serán adiestradas nuevamente y encontrarán trabajos en otras industrias. Pero la política monetaria (y fiscal) no puede capacitar a las personas. La política monetaria (y fiscal) no puede solucionar esos problemas].

Por lo tanto, la combinación de políticas de demanda y oferta aumentará la efectividad y la eficiencia de ambas. Cada una de estas políticas puede fracasar fácilmente: mejorar la mediación en el mercado laboral cuando la falta de demanda total es la razón clave de una crisis difícilmente puede superar el desempleo. En lo que respecta a las políticas de demanda, emerge un segundo "lema de ineficacia política": en altos niveles de desajuste, las medidas expansivas de las políticas monetarias o fiscales conducirán a una mayor inflación en primer lugar con pocas repercusiones (positivas) en la vertiente real de la economía. 


\section{Referencias}

Arellano, M., \& Bond, B. (1991). Some tests of specification for panel data: Monte Carlo evidence and an application to employment equations. Review of Economic Studies, 2(58), 277-297. doi: https://doi.org/10.2307/2297968

Arellano, M., \& Bover, O. (1995). Another look at the instrumental variable estimation of errorcomponents models. Journal of Econometrics, 1(68), 29-51. doi: https://doi.org/10.1016/0304-4076(94)01642-D

Bell, D., \& Blanchflower, D. (2011). Youth Unemployment in Europe and the United States. Institute for the Study of Labor (IZA): Discussion Paper 5673. Recuperado de http://ftp.iza.org/dp5673.pdf

Beveridge, W. (1944). Full Employment in a Free Society. London: The New Statesman and Nation and Reynolds News.

Blanchard, O., \& Diamond, P. A. (1989). The Beveridge Curve. Economic Activity, 1(20), 1-76. Recuperado de https://www.brookings.edu/wpcontent/uploads/1989/01/1989a_bpea_blanchard_diamond hall_yellen.pdf

Blundell, R., \& Bond, S. (1998). Initial conditions and moment restrictions in dynamic panel data models. Journal of Econometrics, 1(87), 115-143. doi: https://doi.org/10.1016/S0304-4076(98)00009-8

Börsch-Supan, A. H. (1991). Panel Data Analysis of the Beveridge Curve, Is There a Macroeconomic Relation between the Rate of Unemployment and the Vacancy Rate? Economica, 231(58), 279-297. doi: https://doi.org/10.2307/2554817

Bouvet, F. (2011). The Beveridge curve in Europe: New evidence using national and regional data. Applied Economics, 27(44), 3.585-3.604. doi: https://doi.org/10.1080/00036846.2011.579062

Capaldo, J., \& Izurieta, A. (2013). The imprudence of labour market flexibilization in a fiscally austere world, International Labour Review, 1(152), 1-26. Recuperado de https://onlinelibrary.wiley.com/doi/abs/10.1111/j.1564-913X.2013.00166.x

Dixon, R., Lim, G. C., \& Freebairn, J. (2012). Regional Beveridge Curves: A Latent Variable Approach. Regional Studies, 2(48), 254-269. doi: https://doi.org/10.1080/00343404.2011.644238 
European Commission. (2012). Labour Market Development in Europe 2012. European Economy 5/2012. Recuperado de http://ec.europa.eu/economy finance/publications/european_economy/2012/2 012-labour-market_en.htm

Fok, D., Franses, P. H., \& Paap, R. (2005). Performance of Seasonal Adjustment Procedures: Simulation and Empirical Results. Report (N. ${ }^{\circ}$ El 2005-30). Recuperado del sitio web de Erasmus University Rotterdam: hdl.handle.net/1765/6917

Haincourt, S., \& Mogliani, M. (2012). Has the 2008-2009 recession increased structural unemployment in the euro area? Quarterly selection of articles, Bulletin de la Banque de France, 25, 63-80. Recuperado de https://publications.banquefrance.fr/sites/default/files/medias/documents/quarterly-selection-ofarticles 25 2012-spring.pdf

Hansen, L. P. (2001). Generalized Method of Moments Estimation: A Time Series Perspective. Recuperado de https://pdfs.semanticscholar.org/d4b9/fccf7f1bdeb0772ecf234ea8cd77ffd386a5 . pdf

Hobijn, B., \& Şahin, A. (2012). Beveridge Curve Shifts across Countries since the Great Recession. https://www.imf.org/external/np/res/seminars/2012/arc/pdf/HS.pdf

Kaufman, B., \& Hotchkiss, J. L. (2006). The Economics of Labor Markets. Recuperado de https://books.google.co.cr/books/about/The Economics of Labor Markets.htm I? id=4ytXKwAACAAJ\&redir esc $=y$

Lacuesta, A., \& Puente, S. (2013). La reforma laboral de 2012: Un primer análisis de algunos de sus efectos sobre el mercado de trabajo. Dirección General del Servicio de Estudios del Banco de España. Recuperado de https://www.bde.es/f/webbde/SES/Secciones/Publicaciones/InformesBoletinesR evistas/BoletinEconomico/13/Sep/Fich/be1309-art5.pdf

Layard, R., Nickell, S., \& Jackman, R. (1994). Unemployment, Macroeconomic Performance and the Labor Market. Inglaterra: Oxford University Press.

Mundlak, Y. (1978). On the Pooling of Time Series and Cross Section Data. Econometrica, 1(46), 69-85. doi: https://doi.org/10.2307/1913646

O'Grady, M. A. (12 de febrero de 2011). The FED's Easy Money Skeptic. Wall Street Journal. Recuperado https://www.wsj.com/articles/SB10001424052748704709304576124132413782 $\underline{592}$ 
Organización para la Cooperación y el Desarrollo Económico. (2013). The 2012 Labour Market Reform in Spain: A Preliminary Assessment. doi: http://dx.doi.org/10.1787/9789264213586-en

Petrongolo, B., \& Pissarides, C. A. (2001). Looking into the Black Box: A Survey of the Matching Function. Journal of Economic Literature, 2(34), 390-431. doi: https://doi.org/10.1257/jel.39.2.390

Phillips, A. W. (1958). The Relation Between Unemployment and the Rate of Change of Money Wage Rates in the UK, 1861-1957. Economica, 100(25), 283-299. doi: https://doi.org/10.1111/j.1468-0335.1958.tb00003.x

Real Decreto-ley 9/2008, Número 290, 48125-48129 (2008). Recuperado de https://www.boe.es/boe/dias/2008/12/02/pdfs/A48125-48130.pdf

Ruesga, S. M., Da Silva, B. y Pérez, M. (2015). La Gran Recesión y sus efectos sobre la eficiencia del mercado laboral. Un estudio aplicado a nivel regional para determinar el mismatch agregado en España. Estudios de Economía Aplicada, 33(1), 175-200. Recuperado de http://www.redalyc.org/articulo.oa?id=30133775009

Sell, F. (2010). Desempleo, desajuste en el mercado laboral ('mismatch') e inflación: Un modelo integrativo (Working Paper). Recuperado del sitio web de ECONSTOR: https://www.econstor.eu/bitstream/10419/32835/1/62575686X.pdf

Sell, F., \& Reinisch, D. C. (2013). How do the Eurozone's Beveridge and Phillips curves perform in the face of global economic crisis? International Labour Review, 2(152), 191204. doi: https://doi.org/10.1111/j.1564-913X.2013.00177.x

Wall, H. J., \& Zoega, G. (2002). The British Beveridge Curve: A Tale of Ten Regions. Oxford Bulletin of Economic and Statistics, 3(64), 257-276. doi: https://doi.org/10.1111/1468-0084.00019

Woo, N. (2012). Urban Myth: Structural Unemployment in Today's Economy. En Blog of Center for Economic and Policy Research (CEPR Blog). Recuperado de http://cepr.net/blogs/cepr-blog/urban-myth-structural-unemployment-intodays-economy

Wölfl, A., \& J. Mora-Sanguinetti. (2011). Reforming the Labour Market in Spain (Working Papers Number 845). Recuperado del sitio web de la Organización para la Cooperación y el Desarrollo Económico. doi: http://dx.doi.org/10.1787/5kghtchh277h-en 